\title{
From Dirac Action to ELKO Action
}

\author{
J. M. Hoff da Silva* \\ Instituto de Física Teórica, Universidade Estadual Paulista, \\ Rua Pamplona 145, 01405-900 São Paulo, SP, Brazil \\ Roldão da Rochàं \\ Centro de Matemática, Computação e Cognição, \\ Universidade Federal do ABC, 09210-170, Santo André, SP, Brazil
}

\begin{abstract}
A fundamental action, representing a mass dimension-transmuting operator between Dirac and ELKO spinor fields, is performed on the Dirac Lagrangian, in order to lead it into the ELKO Lagrangian. Such a dynamical transformation can be seen as a natural extension of the Standard Model that incorporates dark matter fields. The action of the mass dimensiontransmuting operator on a Dirac spinor field, that defines and introduces such a mapping, is shown to be a composition of the Dirac operator and the non-unitary transformation that maps Dirac spinor fields into ELKO spinor fields, defined in [4]. This paper gives allowance for ELKO, as a candidate to describe dark matter, to be incorporated in the Standard Model. It is intended to present for the first time, up to our knowledge, the dynamical character of a mapping between Dirac and ELKO spinor fields, transmuting the mass dimension of spin one-half fermionic fields from $3 / 2$ to 1 and from 1 to $3 / 2$.

PACS numbers: 03.65.Pm, 11.15.-q, 98.80.-k
\end{abstract}

*Electronic address: hoff@ift.unesp.br

${ }^{\dagger}$ Electronic address: roldao.rocha@ufabc.edu.br 


\section{INTRODUCTION}

ELKO spinor fields ${ }^{1}$ are unexpected spin one-half — presenting mass dimension 1 - matter fields, which belong to a non-standard Wigner class [1, 2], and are obtained from a complete set of dual-helicity eigenspinors of the charge conjugation operator. Due to the unusual mass dimension, ELKO spinor fields interact in few possibilities with the Standard Model particles, which instigates it to be a prime candidate to describe dark matter ${ }^{2}$. Indeed, the new matter fields - constructed via ELKO [3] — are dark with respect to the matter and gauge fields of the Standard Model (SM), interacting only with gravity and the Higgs boson [1, 2]. Moreover, it is essential to try to incorporate ELKO spinor fields in some extension of the SM, identifying new fields to dark matter and suggesting how the dark matter sector Lagrangian density arises from a mass dimension-transmuting symmetry.

It was realized in [4] that there exists a non-unitary transformation that can map Dirac spinor fields into ELKO spinor fields, transmuting the mass dimension of spin one-half fermionic fields from $3 / 2$ to 1 - and from 1 to $3 / 2$. The mapping was obtained using algebraic constraints of the Lounesto spinor fields algebraic classification, based upon the bilinear covariants [5, 6, 7], and it was shown that there always exists an invertible operator $M$ such that $M \psi=\lambda$ - and obviously $\psi=M^{-1} \lambda$ - where $\psi$ is a Dirac spinor field and $\lambda$ denotes an ELKO. Also $\bar{\psi}=\lambda^{\dagger}\left(M^{-1}\right)^{\dagger} \gamma^{0}[4]$.

Now we additionaly consider the possibility of incorporating the dynamics of ELKO spinor fields, extending the SM in order to accomplish the dynamical, as well the not less fundamental, algebraic, topological and geometric properties, associated with ELKO. We also emphasize that all the formalism in this paper is exhibited from a classical field theoretical point of view.

This paper is organized as follows: in Section II, ELKO spinor fields are briefly recalled, and in Section III the operator which maps Dirac spinors fields in ELKO, generically called $M$ here, is recalled. For the sake of completeness, there is also an Appendix explaining further topics on the construction of such an operator. In Section IV the mass dimension-transmuting operator $\Theta$ related to the mapping of the Dirac Lagrangian into the ELKO Lagrangian — is introduced, where its action on spinor fields is shown to be the Dirac operator composed with the operator that leads Dirac spinor fields to ELKO. The ELKO Lagrangian is then obtained when a particular class of the operator $\Theta$ is considered, apart from a surface term that can be dismissed when suitable boundary

\footnotetext{
1 The acronym for Eigenspinoren des Ladungskonjugationsoperators or Dual-helicity eigenspinors of the charge conjugation operator [1].

2 Other motivations for the ELKO to be a prime candidate to describe dark matter can be seen in, e.g., [1, 2].
} 
conditions are chosen. In the final Section we conclude summarizing our results, remarking some important points as well as speculating about the possibility of use the $\Theta$ operator in order to map results from Dirac lagrangian into ELKO lagrangian and vice-versa.

\section{ELKO SPINOR FIELDS}

In this Section the formal properties of ELKO spinor fields are briefly revised [1, 2, 8]. An ELKO, denoted by $\Psi$, corresponding to a plane wave with momentum $p=\left(p^{0}, \mathbf{p}\right)$ can be written, without loss of generality, as $\Psi(p)=\lambda(\mathbf{p}) e^{-i p \cdot x}\left(\right.$ or $\Psi(p)=\lambda(\mathbf{p}) e^{i p \cdot x}$ ) where

$$
\lambda(\mathbf{p})=\left(\begin{array}{c}
i \Phi \phi_{L}^{*}(\mathbf{p}) \\
\phi_{L}(\mathbf{p})
\end{array}\right)
$$

$\phi_{L}(\mathbf{p})$ denotes a left-handed Weyl spinor, and given the rotation generators denoted by $\mathfrak{J}$, the Wigner's spin-1/2 time reversal operator $\Phi$ satisfies $\Phi \mathfrak{J} \Phi^{-1}=-\mathfrak{J}^{*}$. Hereon, as in [1], the Weyl representation of $\gamma^{\mu}$ is used, i.e.,

$$
\gamma_{0}=\gamma^{0}=\left(\begin{array}{ll}
\mathbb{O} & \mathbb{I} \\
\mathbb{I} & \mathbb{O}
\end{array}\right), \quad-\gamma_{k}=\gamma^{k}=\left(\begin{array}{cc}
\mathbb{O} & -\sigma_{k} \\
\sigma_{k} & \mathbb{O}
\end{array}\right), \quad \gamma^{5}=-i \gamma^{0} \gamma^{1} \gamma^{2} \gamma^{3}=-i \gamma^{0123}=\left(\begin{array}{cc}
\mathbb{I} & \mathbb{O} \\
\mathbb{O} & -\mathbb{I}
\end{array}\right)
$$

where

$$
\mathbb{I}=\left(\begin{array}{ll}
1 & 0 \\
0 & 1
\end{array}\right), \quad \mathbb{O}=\left(\begin{array}{ll}
0 & 0 \\
0 & 0
\end{array}\right), \quad \sigma_{1}=\left(\begin{array}{ll}
0 & 1 \\
1 & 0
\end{array}\right), \quad \sigma_{2}=\left(\begin{array}{cc}
0 & -i \\
i & 0
\end{array}\right), \quad \sigma_{3}=\left(\begin{array}{cc}
1 & 0 \\
0 & -1
\end{array}\right) .
$$

ELKO spinor fields are eigenspinors of the charge conjugation operator $C$, i.e., $C \lambda(\mathbf{p})= \pm \lambda(\mathbf{p})$, for $C=\left(\begin{array}{cc}\mathbb{O} & i \Phi \\ -i \Phi & \mathbb{O}\end{array}\right) K$. The operator $K$ is responsible for the $\mathbb{C}$-conjugation of spinor fields appearing on the right. The plus sign stands for self-conjugate spinors, $\lambda^{S}(\mathbf{p})$, while the minus yields anti self-conjugate spinors, $\lambda^{A}(\mathbf{p})$. Explicitly, the complete form of ELKO spinor fields can be found by solving the equation of helicity $(\sigma \cdot \widehat{\mathbf{p}}) \phi^{ \pm}(\mathbf{0})= \pm \phi^{ \pm}(\mathbf{0})$ in the rest frame and subsequently performing a boost, in order to recover the result for any p [1]. Note that the helicity of $i \Phi\left[\phi_{L}(\mathbf{p})\right]^{*}$ is opposed to that of $\phi_{L}(\mathbf{p})$, since $(\sigma \cdot \widehat{\mathbf{p}}) \Phi\left[\phi_{L}^{ \pm}(\mathbf{0})\right]^{*}=\mp \Phi\left[\phi_{L}^{* \pm}(\mathbf{0})\right]^{*}$. Here $\widehat{\mathbf{p}}:=\mathbf{p} /\|\mathbf{p}\|=(\sin \theta \cos \phi, \sin \theta \sin \phi, \cos \theta)$. The four spinor fields are given by

$$
\begin{gathered}
\lambda_{\{\mp, \pm\}}^{S / A}(\mathbf{p})=\sqrt{\frac{E+m}{2 m}}\left(1 \mp \frac{\mathbf{p}}{E+m}\right) \lambda_{\{\mp, \pm\}}^{S / A}(\mathbf{0}), \\
\text { where } \quad \lambda_{\{\mp, \pm\}}(\mathbf{0})=\left(\begin{array}{c} 
\pm i \Theta\left[\phi^{ \pm}(\mathbf{0})\right]^{*} \\
\phi^{ \pm}(\mathbf{0})
\end{array}\right) .
\end{gathered}
$$


The phases are adopted so that

$$
\phi^{+}(\mathbf{0})=\sqrt{m}\left(\begin{array}{c}
\cos (\theta / 2) e^{-i \phi / 2} \\
\sin (\theta / 2) e^{i \phi / 2}
\end{array}\right), \quad \phi^{-}(\mathbf{0})=\sqrt{m}\left(\begin{array}{c}
-\sin (\theta / 2) e^{-i \phi / 2} \\
\cos (\theta / 2) e^{i \phi / 2}
\end{array}\right)
$$

at rest, and since $\Theta\left[\phi^{ \pm}(\mathbf{0})\right]^{*}$ and $\phi^{ \pm}(\mathbf{0})$ present opposite helicities, ELKO cannot be an eigenspinor field of the helicity operator, and indeed carries both helicities. In order to guarantee an invariant real norm, as well as positive definite norm for two ELKO spinor fields, and negative definite norm for the other two, the ELKO dual is given by [1]

$$
\lambda_{\{\mp, \pm\}}^{S / A}(\mathbf{p})= \pm i\left[\lambda_{\{ \pm, \mp\}}^{S / A}(\mathbf{p})\right]^{\dagger} \gamma^{0} .
$$

It is useful to choose $i \Theta=\sigma_{2}$, as in [1], in such a way that it is possible to express

$$
\lambda(\mathbf{p})=\left(\begin{array}{c}
\sigma_{2} \phi_{L}^{*}(\mathbf{p}) \\
\phi_{L}(\mathbf{p})
\end{array}\right)
$$

Now, any flagpole spinor field is an eigenspinor field of the charge conjugation operator [6, 7], here represented by $\mathcal{C} \psi=-\gamma^{2} \psi^{*}$. Indeed

$$
-\gamma^{2} \lambda^{*}=\left(\begin{array}{cc}
0 & \sigma_{2} \\
-\sigma_{2} & 0
\end{array}\right)\left(\begin{array}{c}
\left(\sigma_{2} \phi^{*}\right)^{*} \\
\phi^{*}
\end{array}\right)=\left(\begin{array}{c}
\sigma_{2} \phi^{*} \\
-\sigma_{2} \sigma_{2}^{*} \phi
\end{array}\right)=\lambda
$$

\section{MAPPING BETWEEN DIRAC AND ELKO SPINOR FIELDS}

In this Section we briefly review which are the Dirac spinor fields that can be led to ELKO spinor fields. First introduce a matrix $M \in \mathbb{C}(4)$ that defines the transformation from an a priori arbitrary Dirac spinor field to an ELKO spinor field, i.e.,

$$
M \psi=\lambda .
$$

In [4] it was proved that not all DSFs can be led to ELKO, but only a subset of the three classes - under Lounesto classification - of DSFs restricted to some conditions. Explicitly writing the entries of $M=\left[m_{p q}\right]_{p, q=1}^{4}$, we showed in [4] that a general form for $M$ is given by

$$
M=\left(\begin{array}{cccc}
m_{11} & m_{12} & -\chi m_{11} & -i \epsilon \kappa-\chi m_{12} \\
m_{21} & m_{22} & i \epsilon \kappa-\chi m_{21} & -\chi m_{22} \\
m_{31} & m_{32} & 1-\chi m_{31} & -\chi m_{32} \\
m_{41} & m_{42} & -\chi m_{41} & 1-\chi m_{42}
\end{array}\right),
$$


where $\phi_{R}(\mathbf{p})=\chi \phi_{L}(\mathbf{p})$, and $\chi=\frac{E+\sigma \cdot \mathbf{p}}{m}$ and $\kappa \psi=\psi^{*}$. It should be emphasized that $M$ is not unique, and also, in [4] we completely fixed the matrix $M$, inserting the ansatz

$$
\begin{array}{r}
m_{11}=m_{22}=0=m_{32}=m_{41}, \\
m_{31}=m_{42}=1=m_{12}
\end{array}
$$

in such a way that $M$ is written as

$$
M=\left(\begin{array}{cccc}
0 & 1 & 0 & -i \epsilon \kappa-\chi \\
-1 & 0 & i \epsilon \kappa+\chi & 0 \\
1 & 0 & 1-\chi & 0 \\
0 & 1 & 0 & 1-\chi
\end{array}\right)
$$

Note that such matrix is not unitary, and since $\operatorname{det} M \neq 0$, there exists (see Eq.(9) ) $M^{-1}$ such that $\psi=M^{-1} \lambda$. Besides, it is immediate to note that

$$
\bar{\psi}:=\psi^{\dagger} \gamma^{0}=\lambda^{\dagger}\left(M^{-1}\right)^{\dagger} \gamma^{0}
$$

such that $\bar{\psi}$ can be related to the ELKO dual by

$$
\bar{\psi}=\mp i \lambda_{\{\mp, \pm\}}^{S / A} \gamma^{0}\left(M^{-1}\right)^{\dagger} \gamma^{0}
$$

In what follows, the matrix $M$ establishes necessary conditions on the Dirac spinor fields under which the mapping given by Eq.(9) is satisfied. However, the ansatz in Eq.(12) has just an illustrative rôle. In fact, for any matrix satisfying Eq.(10), there are corresponding constraints on the components of DSFs. Hereafter, we shall calculate the conditions to the case where $\mathbf{p}=0$ (and consequently $\chi=1$ ), since a Lorentz boost can be implemented on the rest frame in the constraints. Anyway, without lost of generality, the conditions to be found on DSFs must hold in all referentials, and in particular in the rest frame corresponding to $\mathbf{p}=0$.

Denoting the Dirac spinor field as $\psi=\left(\psi_{1}, \psi_{2}, \psi_{3}, \psi_{4}\right)^{T}\left(\psi_{r} \in \mathbb{C}, r=1, \ldots, 4\right)$, we achieved simultaneous conditions for the Dirac spinor field be led to an ELKO spinor field respectively:

$$
\begin{aligned}
& 0=\operatorname{Re}\left(\psi_{1}^{*} \psi_{3}\right)+\operatorname{Re}\left(\psi_{2}^{*} \psi_{4}\right) \\
& 0=\operatorname{Re}\left(\psi_{2}^{*} \psi_{3}\right)+\operatorname{Re}\left(\psi_{1}^{*} \psi_{4}\right) \\
& 0=\operatorname{Im}\left(\psi_{1}^{*} \psi_{4}\right)-\operatorname{Im}\left(\psi_{2}^{*} \psi_{3}\right)-2 \operatorname{Im}\left(\psi_{3}^{*} \psi_{4}\right)-2 \operatorname{Im}\left(\psi_{1}^{*} \psi_{2}\right) \\
& 0=\operatorname{Re}\left(\psi_{1}^{*} \psi_{3}\right)-\operatorname{Re}\left(\psi_{2}^{*} \psi_{4}\right) .
\end{aligned}
$$


Note that the first and the last conditions together mean $\mathbb{R e}\left(\psi_{1}^{*} \psi_{3}\right)=0$ and $\mathbb{R e}\left(\psi_{2}^{*} \psi_{4}\right)=0$. In what follows we obtain the extra necessary and sufficient conditions for each class of Dirac spinor fields, according to Lounesto spinor fields classification (See Appendix).

Using the decomposition $\psi_{j}=\psi_{j a}+i \psi_{j b}$ (where $\psi_{j a}=\mathbb{R e}\left(\psi_{j}\right)$ and $\left.\psi_{j b}=\operatorname{Im}\left(\psi_{j}\right)\right)$ it follows that $\mathbb{R e}\left(\psi_{i}^{*} \psi_{j}\right)=\psi_{i a} \psi_{j a}+\psi_{i b} \psi_{j b}$ and $\operatorname{Im}\left(\psi_{i}^{*} \psi_{j}\right)=\psi_{i a} \psi_{j b}-\psi_{i b} \psi_{j a}$ for $i, j=1, \ldots, 4$. So, in components, the conditions in common for all types of DSFs are

$$
\begin{aligned}
& \psi_{1 a} \psi_{3 a}+\psi_{1 b} \psi_{3 b}=0, \\
& \psi_{2 a} \psi_{4 a}+\psi_{2 b} \psi_{4 b}=0,
\end{aligned}
$$

and the additional conditions for each case are summarized in Table I below.

\begin{tabular}{|c|c|}
\hline Class & Additional conditions \\
\hline \hline$(1)$ & $\psi_{2 a}\left(\psi_{3 a}-\psi_{3 b}\right)+\psi_{2 b}\left(\psi_{3 a}+\psi_{3 b}\right)=0=\psi_{3 a} \psi_{4 b}-\psi_{3 b} \psi_{4 a}$ \\
\hline$(2)$ & $\psi_{3 a} \psi_{4 b}-\psi_{3 b} \psi_{4 a}=0=\psi_{2 a} \psi_{3 a}+\psi_{2 b} \psi_{3 b}+\psi_{1 a} \psi_{4 a}+\psi_{1 b} \psi_{4 b}$ \\
\hline$(3)$ & $\psi_{2 a}\left(\psi_{3 a}-\psi_{3 b}\right)+\psi_{2 b}\left(\psi_{3 a}+\psi_{3 b}\right)=0$ and \\
& $\left(\psi_{1 a} \psi_{4 b}-\psi_{1 b} \psi_{4 a}\right)-\left(\psi_{2 a} \psi_{3 b}-\psi_{2 b} \psi_{3 a}\right)-2\left(\psi_{3 a} \psi_{4 b}-\psi_{3 b} \psi_{4 a}\right)-2\left(\psi_{1 a} \psi_{2 b}-\psi_{1 b} \psi_{2 a}\right)=0$ \\
\hline
\end{tabular}

TABLE I: Additional conditions, in components, for class (1), (2) and (3) Dirac spinor fields.

Once the matrix $M$ - leading an arbitrary Dirac spinor field to an ELKO - has been introduced, we proved that it can be written in the general form given by Eq.(10), without loss of generality. The ansatz given by Eq.(10) is useful to illustrate and explicitly exhibit how to obtain the necessary conditions on the components of a DSF — under Lounesto spinor field classification (see Appendix, for more details and references therein - in order to it be led to an ELKO spinor field. In the case of a type-(1) Dirac spinor field there are six conditions, and then the equivalence class of type-(1) Dirac spinor fields that can be led to ELKO spinor fields can be written in the form $^{3}$

$$
\psi=\left(\begin{array}{c}
\psi_{1} \\
f_{1}\left(\psi_{1}\right) \\
f_{2}\left(\psi_{1}\right) \\
f_{3}\left(\psi_{1}\right)
\end{array}\right)
$$

\footnotetext{
${ }^{3}$ Among the three equivalent definitions of spinor fields, viz., the classical, algebraic, and operatorial, here the classical one - where a spinor is an element that carries the representation space of the group $\operatorname{Spin}_{+}(1,3)$, is regarded.
} 
where $f_{i}$ are complex scalar functions of the component $\psi_{1} \in \mathbb{C}$ of $\psi$, obtainable - using the Implicit Function Theorem - through the conditions given in Eqs.(16), (17), and also those given by Table I. For a general and arbitrary ansatz, the equivalence class of type-(1) DSFs that can be led to ELKO spinor fields, via the matrix $M$, are given by

$$
\psi=\left(\begin{array}{c}
\psi_{1} \\
g_{1}(M)\left(\psi_{1}\right) \\
g_{2}(M)\left(\psi_{1}\right) \\
g_{3}(M)\left(\psi_{1}\right)
\end{array}\right)
$$

where each $g_{i}(M)$ is a complex scalar function of the component $\psi_{1} \in \mathbb{C}$ of $\psi$. Such scalar functions depend explicitly on the form of $M$, and to a fixed but arbitrary $M$ there corresponds other six conditions analogous to Eqs.(16), (17), and also those given by Table I. All these conditions obtained by the ansatz is general, and illustrates the general procedure of finding the conditions. For the equivalence class of type-(2) and -(3) DSFs that are led to ELKO spinor fields, it is only demanded five conditions, instead of six [4]. In both cases, the most general form of the DSFs are given by

$$
\psi=\left(\begin{array}{c}
\psi_{1 a}+i \psi_{1 b} \\
\psi_{2 a}+i \psi_{2 b} \\
\psi_{3 a}+i \psi_{3 b} \\
\psi_{4 a}+i \psi_{4 b}
\end{array}\right)=\left(\begin{array}{c}
\psi_{1 a}+i \psi_{1 b} \\
\psi_{2 a}+i h_{1}(M)\left(\psi_{1 a}, \psi_{1 b}, \psi_{2 a}\right) \\
h_{2}(M)\left(\psi_{1 a}, \psi_{1 b}, \psi_{2 a}\right)+i h_{3}(M)\left(\psi_{1 a}, \psi_{1 b}, \psi_{2 a}\right) \\
h_{4}(M)\left(\psi_{1 a}, \psi_{1 b}, \psi_{2 a}\right)+i h_{5}(M)\left(\psi_{1 a}, \psi_{1 b}, \psi_{2 a}\right)
\end{array}\right)
$$

where each $h_{A}(M)(A=1, \ldots, 5)$ is a $M$ matrix-dependent real scalar function of the (real) components $\psi_{1 a}, \psi_{1 b}, \psi_{2 a}$ of $\psi$. For more details and considerations see [4].

To summarize, the development reviewed in this Section is accomplished without any loss of generality. For more details and considerations see [4]. In the next Section we shall to introduce an operator, constructed upon $M$, responsible to lead the Dirac action into the ELKO action, apart from a surface term.

\section{MAPPING THE DIRAC LAGRANGIAN IN THE ELKO LAGRANGIAN}

In this Section the action of the mass dimension-transmuting $\Theta$ operator on the Dirac Lagrangian is explicitly derived, leading to the ELKO Lagrangian plus a surface term. When the transformation of the spinor fields constituting the Lagrangian is dealt, there are two possibilities to construct the mass dimension-transmuting $\Theta$ operator action: the fundamental $\left(\Theta \mathcal{L}_{\text {Dirac }}\right)$ and

the adjoint action $\left(\Theta \mathcal{L}_{\text {Dirac }} \Theta^{-1}\right)$. From a formal viewpoint, it is always possible to define the 
adjoint action $\left(\Theta \mathcal{L}_{\text {Dirac }} \Theta^{-1}\right)$, resulting in

$$
\begin{aligned}
\Theta \mathcal{L}_{\text {Dirac }} \Theta^{-1} & =\Theta\left[\left(\bar{\psi} \gamma^{\mu} \partial_{\mu} \psi\right)-m_{D}(\bar{\psi} \psi)\right] \Theta^{-1} \\
& =\Theta \bar{\psi} \Theta^{-1} \Theta \gamma^{\mu} \Theta^{-1} \Theta \partial_{\mu} \psi \Theta^{-1}-m_{D} \Theta \bar{\psi} \Theta^{-1} \Theta \psi \Theta^{-1}
\end{aligned}
$$

There is no reason a priori for fixing one of the two actions, although the adjoint action resembles some correspondent physical symmetry, in the case it is performed by an unitary operator. But it can be shown that there is not an unitary operator able to lead the Dirac Lagrangian to the ELKO Lagrangian, and the Dirac and ELKO spinor fields present respectively local and non-local properties. It follows immediately from the results in [1, 4].

If $\Theta$ does exist as a true physical operator, the extended Lagrangian density describes the SM in order to incorporate dark matter fields [3]. Hereon the fundamental action is taken into account, by defining an (even) derivation $\Theta$ on the space of spinor fields $(\Theta(\psi \phi)=(\Theta \psi) \phi+\psi(\Theta \phi)$, where $\psi, \phi$ denote spinor fields) such that $\Theta i=i \Theta$. Such an operator acts on $\mathcal{L}_{\text {Dirac }}$ as

$$
\begin{aligned}
\Theta \mathcal{L}_{\text {Dirac }} & =\Theta\left(i \bar{\psi} \gamma^{\mu} \partial_{\mu} \psi-m_{D} \bar{\psi} \psi\right) \\
& =i \Theta\left(\bar{\psi} \gamma^{\mu} \partial_{\mu} \psi\right)-m_{D} \Theta(\bar{\psi} \psi) \\
& =i\left[(\Theta \bar{\psi}) \gamma^{\mu} \partial_{\mu} \psi+\bar{\psi} \Theta\left(\gamma^{\mu} \partial_{\mu} \psi\right)\right]-m_{D}[\Theta(\bar{\psi}) \psi+\bar{\psi}(\Theta \psi)]
\end{aligned}
$$

By straightforward algebraic manipulation, this equation can be expressed as

$$
\begin{aligned}
\Theta \mathcal{L}_{\text {Dirac }} & =(\Theta \bar{\psi})\left(i \not \partial-m_{D}\right) \psi+i \bar{\psi}\left(\Theta \gamma^{\mu}\right) \partial_{\mu} \psi+i \bar{\psi} \gamma^{\mu} \partial_{\mu}(\Theta \psi)-i \bar{\psi} \gamma^{\mu}\left(\partial_{\mu} \Theta\right) \psi-m_{D} \bar{\psi}(\Theta \psi) \\
& =(\Theta \bar{\psi})\left(i \not \partial-m_{D}\right) \psi+\bar{\psi}\left(i \not \partial-m_{D}\right)(\Theta \psi)+i \bar{\psi}\left(\Theta \gamma^{\mu}\right) \partial_{\mu} \psi-i \bar{\psi} \gamma^{\mu}\left(\partial_{\mu} \Theta\right) \psi
\end{aligned}
$$

where $\not \partial=\gamma^{\mu} \partial_{\mu}$.

Now, we construct the spinor space derivation $\Theta$ satisfying the required property $\Theta i=i \Theta$ in such a way that its action on $\bar{\psi}$ is given by

$$
\Theta \bar{\psi}=-\frac{m^{2}}{2} \bar{\psi} \gamma^{0} M^{\dagger} i \gamma^{0} M\left(i \not \partial-m_{D}\right)^{-1}
$$

where $M$ is the matrix introduced in the previous Section. Note that the nontrivial form of Eq.(24) is motivated and justified by the property

$$
\begin{aligned}
(\Theta \bar{\psi})\left(i \not \partial-m_{D}\right) \psi & =-\frac{m^{2}}{2} \bar{\psi} \gamma^{0} M^{\dagger} i \gamma^{0} M \psi \\
& =-\frac{m^{2}}{2} \lambda^{\dagger}\left(M^{-1}\right)^{\dagger} \gamma^{0} \gamma^{0} M^{\dagger} i \gamma^{0} M M^{-1} \lambda \\
& =-\frac{m^{2}}{2} \vec{\lambda} \lambda
\end{aligned}
$$


which is exactly half the mass term in ELKO Lagrangian [1, 2, 8]. The mass dimension associated with the $m_{D}$ parameter equals the mass dimension of $m$. We shall, however, preserve this notation in order to avoid confusion. In Eq.(25) we used the fact that $M \psi=\lambda$ and $\psi=M^{-1} \lambda$, where $\psi$ is a Dirac spinor field and $\lambda$ denotes an ELKO [4]. Also $\bar{\psi}=\lambda^{\dagger}\left(M^{-1}\right)^{\dagger} \gamma^{0}$. In addition, it is possible to apply the operator $\Theta$, satisfying the same required property $\Theta i=i \Theta$, on the Dirac dual spinor field in such a way that

$$
\Theta \psi=-\left(i \not \partial-m_{D}\right)^{-1} \frac{m^{2}}{2} \gamma^{0} M^{\dagger} i \gamma^{0} M \psi
$$

and, in a similar reasoning related to the derivation of Eq.(25), the mass term in ELKO Lagrangian is given by $\bar{\psi}\left(i \not \partial-m_{D}\right) \Theta \psi=-\frac{m^{2}}{2} \vec{\lambda} \lambda$. The action of the symmetry operator $\Theta$ on the Dirac Lagrangian can be expressed, using Eq.(23), as

$$
\Theta \mathcal{L}_{\operatorname{Dirac}}=i \bar{\psi}\left[\left(\Theta \gamma^{\mu}\right) \partial_{\mu}-\gamma^{\mu}\left(\partial_{\mu} \Theta\right)\right] \psi-m^{2} \vec{\lambda} \lambda
$$

The $\Theta$ operator always exists, in the sense that it is constructed in terms of the Dirac operator derivatives, of the Dirac matrices, and also in terms of $M$. As these three objects always exist, $\Theta$ also always exists. Since $M$ is not unique - it is given by Eq.(10) - so the operator $\Theta$ is also not unique. But once we fix the operator $M$, as in Eq.(12), the operator $\Theta$ is automatically unique.

On the other hand, the general form of the ELKO Lagrangian ${ }^{4}$ is given by the expression [2]

$$
\mathcal{L}_{\mathrm{ELKO}}=\partial_{\mu} \vec{\lambda} \partial^{\mu} \lambda-m^{2} \vec{\lambda} \lambda
$$

Since the transformed Dirac Lagrangian presents the same mass term as the ELKO Lagrangian [2], it is possible to relate the two Lagrangians, using the relations between ELKO and Dirac spinors fields, as

$$
\Theta \mathcal{L}_{\text {Dirac }}=i \bar{\psi}\left[\left(\Theta \gamma^{\mu}\right) \partial_{\mu}-\gamma^{\mu}\left(\partial_{\mu} \Theta\right)\right] \psi+\mathcal{L}_{\mathrm{ELKO}}-\partial_{\mu} \vec{\lambda} \partial^{\mu} \lambda
$$

Integrating by parts, and using the relations $\psi=M^{-1} \lambda, \bar{\psi}=\lambda^{\dagger}\left(M^{-1}\right)^{\dagger} \gamma^{0}$, and Eq.(7), it reads

$$
\Theta \mathcal{L}_{\text {Dirac }}=\mathcal{L}_{\text {ELKO }}-\partial_{\mu}\left(\vec{\lambda} \partial^{\mu} \lambda\right)+\vec{\lambda}\left[\left(\gamma^{0}\left(M^{-1}\right)^{\dagger} \gamma^{0}\left[\left(\Theta \gamma^{\mu}\right) \partial_{\mu}-\gamma^{\mu}\left(\partial_{\mu} \Theta\right)\right] M^{-1}\right)+\square\right] \lambda .
$$

This remarkable expression shows, in the scope illustrated above, a strong relationship between $\Theta \mathcal{L}_{\text {Dirac }}$ and $\mathcal{L}_{\text {ELKO. The }}$ Thecond term in Eq.(30) is a surface term and can be neglected by appropriate boundary conditions. Consequently, it immediately follows from Eq.(30) that

$$
\int \Theta \mathcal{L}_{\text {Dirac }} d^{4} x=\int \mathcal{L}_{\text {ELKO }} d^{4} x+\int \vec{\lambda}\left[\left(\gamma^{0}\left(M^{-1}\right)^{\dagger} \gamma^{0}\left[\left(\Theta \gamma^{\mu}\right) \partial_{\mu}-\gamma^{\mu}\left(\partial_{\mu} \Theta\right)\right] M^{-1}\right)+\square\right] \lambda d^{4} x
$$

\footnotetext{
${ }^{4}$ Without taking into account auto-interaction terms [2].
} 
Let us define the $\mathcal{P}$ operator as

$$
\mathcal{P}=\gamma^{0}\left(M^{-1}\right)^{\dagger} \gamma^{0}(\not \partial \cdot \Theta) M^{-1}+\square
$$

where $\not \partial \cdot \Theta=\left(\Theta \gamma^{\mu}\right) \partial_{\mu}-\gamma^{\mu}\left(\partial_{\mu} \Theta\right)$. Here, given the spacetime metric $g$, the left contraction is implicitly defined by $g(a \cdot b, c)=g(b, \tilde{a} \wedge c)$, where $a, b, c$ denote elements of the Dirac algebra and $\tilde{a}$ denotes the reversion anti-automorphism on the Dirac algebra element $a$ [4, 6]. In addition, $a \wedge b$ denotes the exterior product between two elements $a, b$ in the Dirac algebra. Now, Eq.(31) is suitable written as

$$
\int \Theta \mathcal{L}_{\text {Dirac }} d^{4} x=\int \mathcal{L}_{\text {ELKO }} d^{4} x+\int \vec{\lambda} \mathcal{P} \lambda d^{4} x
$$

In order to define the action $\Theta$ that is responsible to map $\mathcal{L}_{\text {Dirac }}$ to $\mathcal{L}_{\text {ELKO }}$, we must restrict the action $\Theta$ on the spinor space, eliminating then the extra term given by $\int \vec{\lambda} \mathcal{P} \lambda d^{4} x$ in Eq. (333). From Eq.(32), by imposing $\mathcal{P} \equiv 0$, it follows that

$$
\gamma^{0}\left(M^{-1}\right)^{\dagger} \gamma^{0}(\not \partial \cdot \Theta) M^{-1}=-\square
$$

which can be expressed by

$$
\begin{aligned}
(\not \partial \cdot \Theta) & =-\gamma^{0} M^{\dagger} \gamma^{0} \square M \\
& =\left(\gamma^{0} M^{\dagger} i \gamma^{0} M\right) M^{-1} i \square M
\end{aligned}
$$

and, also, using the most general form of the matrix $M$ that leads a Dirac spinor field to an ELKO and presents the properties $M i=i M^{*}, i M=M^{*} i[4]$, Eq.(35) reads

$$
(\not \partial \cdot \Theta)=\left(\gamma^{0} M^{\dagger} i \gamma^{0} M\right) M^{-1} \square M^{*} i \text {. }
$$

The equation above is an additional formal constraint which must be respected by the $\Theta$ operator in order to eliminate the contribution from the $\mathcal{P}$ operator. In order to relate this constraint to the action of $\Theta$ in $\psi$ and $\bar{\psi}$, note that Eqs.(24) and (26) can be respectively written as

$$
(\Theta \bar{\psi})\left(i \not \partial-m_{D}\right)=-\frac{m^{2}}{2} \bar{\psi}\left(\gamma^{0} M^{\dagger} i \gamma^{0} M\right)
$$

and

$$
\left(i \not \partial-m_{D}\right)(\Theta \psi)=-\frac{m^{2}}{2}\left(\gamma^{0} M^{\dagger} i \gamma^{0} M\right) \psi .
$$

Now, using Eq.(36), the action of the operator $\Theta$ on the spinor space arises from Eqs.(37) and (38), and completely defines the action of the symmetry $\Theta$ in the Dirac Lagrangian, in order to lead it to the ELKO Lagrangian. It is given explicitly by

$$
\Theta \bar{\psi}=i \frac{m^{2}}{2} \bar{\psi}\left[(\not \partial \cdot \Theta)\left(\square M^{*}\right)^{-1} M\right]\left(i \not \partial-m_{D}\right)^{-1}
$$


and

$$
\Theta \psi=i \frac{m^{2}}{2}\left(i \not \partial-m_{D}\right)^{-1} \bar{\psi}\left[(\not \partial \cdot \Theta)\left(\square M^{*}\right)^{-1} M\right] \psi
$$

We emphasize that the $(\not \partial \cdot \Theta)$ term appearing in the formulae above is fixed by Eq.(36) in terms of the matrix $M$ that turns Dirac to ELKO spinor fields [4].

After all, from Eq.(31) it is easy to see that

$$
\int\left(\Theta \mathcal{L}_{\text {Dirac }}\right) d^{4} x=\int \mathcal{L}_{\text {ELKO }} d^{4} x
$$

In this form, the operator $\Theta$ (acting according to (39), (40) and (36)) is responsible to lead the Dirac spinor field lagrangian to the ELKO lagrangian, which suggests the possibility of extending the Standard Model of elementary particles in order to incorporate dark matter. We shall to make some comments about this results in the next Section.

\section{CONCLUDING REMARKS AND OUTLOOKS}

We have constructed an operator $\Theta$ intended to transmute the spinor fields which have mass dimension 1 to spinor fields presenting mass dimension 3/2, and vice-versa. It is obtained via the appropriate definition of the $\Theta$ operator acting as an even derivation on the spinor space. This action of the $\Theta$ operator upon the Dirac Lagrangian is intended to transform all the dynamics of a free Dirac spinor field into an also free ELKO spinor field in the classical field theoretical framework. Besides, we have shown that in order to the Dirac Lagrangian to be suitably led to the ELKO Lagrangian, the action of the $\Theta$ operator, defined on both spinors $\psi$ and conjugate spinor $\bar{\psi}$, must obey an additional constraint given by Eq.(36). The actions Eqs.(39) and (40) constitute a minimal set that completely defines the operator $\Theta$, and although it seems $\Theta \psi$ can be written in terms of $\Theta \bar{\psi}$, as the unique difference between them is the common terms $\left[(\not \partial \cdot \Theta)\left(\square M^{*}\right)^{-1} M\right]$ and $\left(i \not \partial-m_{D}\right)^{-1}$ appearing in reverse order, each of these two terms does not possess a definite homogeneous multivectorial structure in the spacetime Dirac algebra. Consequently, if we attempt

to express, e.g., $\Theta \psi$ in terms of $\Theta \bar{\psi}$, both the terms $\left[(\not \partial \cdot \Theta)\left(\square M^{*}\right)^{-1} M\right]$ and $\left(i \not \partial-m_{D}\right)^{-1}$ are modified, when the anti-automorphism that reverses the multivectorial structure in the spacetime Dirac algebra acts on their product. The reversion changes the order of these terms, and in addition modifies each one of the terms $\left[(\not \partial \cdot \Theta)\left(\square M^{*}\right)^{-1} M\right]$ and $\left(i \not \partial-m_{D}\right)^{-1}$.

We should point out a few more remarks about the future of this line of research. First, in the scope of the formalism developed in this paper, the constraint (36) is just a pure formal 
restriction, and does not have an immediate physical interpretation yet. A deeper investigation on such a constraint can reveal some important physical implication about a possible extension of the Standard Model. Second, the transformation analyzed here was obtained in the scope of classical field theory. In quantum field language, ELKO spinor fields are extended objects, while Dirac spinor fields belongs to a standard Wigner's class [1, 2]. The quantum version of the $\Theta$ symmetry should stress this fact. From the functional form of $\Theta$ acting in $\psi$ and $\bar{\psi}$ we see the presence of an object that, in quantum field theory, is identified to the Dirac propagator. This may suggest that the non-local character of the ELKO spinor field is already taken into account. However, obviously, the transformation of a local quantum object into a non-local one brings a deep modification of the canonical structure of the theory. Regarding the formalism developed here, we prefer to be more conservative and not to relate the form of the $\Theta$ operator to any "quantum adjective".

We should emphasize however that, even in the scope of classical field theory, the main results of this paper can be used in order to map solutions from the Dirac lagrangian to ELKO lagrangian and vice-versa, respecting the conditions under which the actions of both systems are related. It seems that, again, the formal constraint (36) plays a crucial role in such a map. Finally, the study of the solutions that can be led from one system to another seems to provide an useful field of research, since the ELKO spinor field is, in many aspects, more tractable than the Dirac spinor field. On the other hand, in taking this program seriously, the vast literature concerning DSF's can bring a lot of physical information about ELKO spinor field itself.

As a last remark, the formalism developed in Section IV can be immediately applied to the dark matter candidate described in [3]. There are proposed new fields describing dark matter and suggesting how the dark matter sector Lagrangian density arises from the mass dimensiontransmuting symmetry $\Theta$, here investigated, constructed upon the algebraic mapping of Dirac spinor fields into ELKO spinor fields. It is one more contribution in order to extend the Standard Model to incorporate dark matter.

In particular, there has a lot of advances accomplished by the refs. [1, 2, 8, 9] where there has been explored the relationship between a quantum field theory related to ELKO spinor fields and some applications in an attempt to describe and investigate dark matter. Once a prescription between Dirac theory and ELKO is accomplished, it could be easier and useful to investigate some properties of dark matter formalism, using the Dirac formalism for relativistic quantum mechanics and quantum field theory. In addition, using the mapping between ELKO spinor fields and the three classes Dirac spinor fields, it was shown in [12] that the Einstein-Hilbert, the EinsteinPalatini, and the Holst actions can be derived from the Quadratic Spinor Lagrangian — used 
as the prime Lagrangian for supergravity. The Holst action is related to the Ashtekar's quantum gravity formulation, and shows up also in the proof of gravitational theory as a SUSY gauge theory as well [13]. To each one of these classes, there corresponds a unique kind of action for a covariant gravity theory [14].

There is a lot of developments based on the present formalism of ELKO action and the Dirac action that can bring some new light on some related research lines.

\section{ACKNOWLEDGMENT}

The authors are very grateful to Prof. Dharamvir Ahluwalia for important comments about this paper, and to the IJMPA Referee as well. Roldão da Rocha thanks to Fundação de Amparo à Pesquisa do Estado de São Paulo (FAPESP) (2008/06483-5) and J. M. Hoff da Silva thanks to CAPES-Brazil for financial support.

\section{Appendix: Bilinear Covariants, Lounesto Spinor Field Classification and ELKO spinor fields}

This Appendix is devoted to recall the bilinear covariants, using the programme introduced in [5], just for book keeping purposes. In this article all spinor fields live in Minkowski spacetime $\left(M, \eta, D, \tau_{\eta}, \uparrow\right)$. The manifold $M \simeq \mathbb{R}^{4}, \eta$ denotes a constant metric, where $\eta\left(\partial / \partial x^{\mu}, \partial / \partial x^{\nu}\right)=$ $\eta_{\mu \nu}=\operatorname{diag}(1,-1,-1,-1), D$ denotes the Levi-Civita connection associated with $\eta, M$ is oriented by the 4 -volume element $\tau_{\eta}$ and time-oriented by $\uparrow$. Here $\left\{x^{\mu}\right\}$ denotes global coordinates in the Einstein-Lorentz gauge, naturally adapted to an inertial reference frame $\mathbf{e}_{0}=\partial / \partial x^{0}$. Let $\mathbf{e}_{i}=\partial / \partial x^{i}, i=1,2,3$. Also, $\left\{\mathbf{e}_{\mu}\right\}$ is a section of the frame bundle $\mathbf{P}_{\mathrm{SO}_{1,3}^{e}}(M)$ and $\left\{\mathbf{e}^{\mu}\right\}$ is its reciprocal frame satisfying $\eta\left(\mathbf{e}^{\mu}, \mathbf{e}_{\nu}\right):=\mathbf{e}^{\mu} \cdot \mathbf{e}_{\nu}=\delta_{\nu}^{\mu}$. Classical spinor fields carrying a $D^{(1 / 2,0)} \oplus D^{(0,1 / 2)}$, or $D^{(1 / 2,0)}$, or $D^{(0,1 / 2)}$ representation of $\operatorname{SL}(2, \mathbb{C}) \simeq \operatorname{Spin}_{1,3}^{e}$ are sections of the vector bundle $\mathbf{P}_{\operatorname{Spin}_{1,3}^{e}}(M) \times{ }_{\rho} \mathbb{C}^{4}$, where $\rho$ stands for the $D^{(1 / 2,0)} \oplus D^{(0,1 / 2)}\left(\right.$ or $D^{(1 / 2,0)}$ or $\left.D^{(0,1 / 2)}\right)$ representation of $\mathrm{SL}(2, \mathbb{C}) \simeq \operatorname{Spin}_{1,3}^{e}$ in $\mathbb{C}^{4}$. Given a spinor field $\psi \in \sec \mathbf{P}_{\operatorname{Spin}_{1,3}^{e}}(M) \times{ }_{\rho} \mathbb{C}^{4}$ the bilinear covariants are the following sections of the exterior algebra bundle of multivector fields [11]:

$$
\begin{aligned}
& \sigma=\psi^{\dagger} \gamma_{0} \psi, \quad \mathbf{J}=J_{\mu} \mathbf{e}^{\mu}=\psi^{\dagger} \gamma_{0} \gamma_{\mu} \psi \mathbf{e}^{\mu}, \quad \mathbf{S}=S_{\mu \nu} \mathbf{e}^{\mu \nu}=\frac{1}{2} \psi^{\dagger} \gamma_{0} i \gamma_{\mu \nu} \psi \mathbf{e}^{\mu} \wedge \mathbf{e}^{\nu}, \\
& \mathbf{K}=\psi^{\dagger} \gamma_{0} i \gamma_{0123} \gamma_{\mu} \psi \mathbf{e}^{\mu}, \quad \omega=-\psi^{\dagger} \gamma_{0} \gamma_{0123} \psi .
\end{aligned}
$$

The set $\left\{\gamma_{\mu}\right\}$ refers to the Dirac matrices in chiral representation (see Eq.(2)). Also $\left\{\mathbf{1}_{4}, \gamma_{\mu}, \gamma_{\mu} \gamma_{\nu}, \gamma_{\mu} \gamma_{\nu} \gamma_{\rho}, \gamma_{0} \gamma_{1} \gamma_{2} \gamma_{3}\right\}(\mu, \nu, \rho=0,1,2,3$, and $\mu<\nu<\rho)$ is a basis for $\mathbb{C}(4)$ satisfy- 
ing [6] $\gamma_{\mu} \gamma_{\nu}+\gamma_{\nu} \gamma_{\mu}=2 \eta_{\mu \nu} \mathbf{1}_{4}$ and the Clifford product is denoted by juxtaposition. More details on notations can be found in [11, 15].

Given a fixed spin frame the bilinear covariants are considered as being the following operator fields, for each $x \in M$, as mappings $\mathbb{C}^{4} \rightarrow \mathbb{C}^{4}$ :

$$
\begin{aligned}
& \sigma=\psi^{\dagger} \gamma_{0} \psi, \quad \mathbf{J}=J_{\mu} \gamma^{\mu}=\psi^{\dagger} \gamma_{0} \gamma_{\mu} \psi \gamma^{\mu}, \quad \mathbf{S}=S_{\mu \nu} \gamma^{\mu \nu}=\frac{1}{2} \psi^{\dagger} \gamma_{0} i \gamma_{\mu \nu} \psi \gamma^{\mu \nu}, \\
& \mathbf{K}=\psi^{\dagger} \gamma_{0} i \gamma_{0123} \gamma_{\mu} \psi \gamma^{\mu}, \quad \omega=-\psi^{\dagger} \gamma_{0} \gamma_{0123} \psi
\end{aligned}
$$

In the case of the electron, described by Dirac spinor fields (classes 1, 2 and 3 below), $\mathbf{J}$ is a futureoriented timelike current vector which gives the current of probability, the bivector $\mathbf{S}$ is associated with the distribution of intrinsic angular momentum, and the spacelike vector $\mathbf{K}$ is associated with the direction of the electron spin. For a detailed discussion concerning such entities, their relationships and physical interpretation, and generalizations, see, e.g., [6, 7, 16, 17, 18].

The bilinear covariants satisfy the Fierz identities [6, 7], 16, 17, 18]

$$
\mathbf{J}^{2}=\omega^{2}+\sigma^{2}, \quad \mathbf{K}^{2}=-\mathbf{J}^{2}, \quad \mathbf{J}\left\llcorner\mathbf{K}=0, \quad \mathbf{J} \wedge \mathbf{K}=-\left(\omega+\sigma \gamma_{0123}\right) \mathbf{S} .\right.
$$

A spinor field such that not both $\omega$ and $\sigma$ are null is said to be regular. When $\omega=0=\sigma$, a spinor field is said to be singular.

Lounesto spinor field classification is given by the following spinor field classes [6, 7], where in the first three classes it is implicit that $\mathbf{J}, \mathbf{K}, \mathbf{S} \neq 0$ :

1) $\sigma \neq 0, \quad \omega \neq 0$

2) $\sigma \neq 0, \quad \omega=0$.

3) $\sigma=0, \quad \omega \neq 0$.

4) $\sigma=0=\omega, \quad \mathbf{K} \neq 0, \quad \mathbf{S} \neq 0$.

5) $\sigma=0=\omega, \quad \mathbf{K}=0, \quad \mathbf{S} \neq 0$.

6) $\sigma=0=\omega, \quad \mathbf{K} \neq 0, \quad \mathbf{S}=0$.

The current density $\mathbf{J}$ is always non-zero. Types-(1), -(2), and -(3) spinor fields are denominated Dirac spinor fields for spin-1/2 particles and types-(4), -(5), and -(6) are respectively called flagdipole [10], flagpole ${ }^{5}$ and Weyl spinor fields. Majorana spinor fields are a particular case of a

5 Such spinor fields are constructed by a null 1-form field current and an also null 2-form field angular momentum, the "flag" [19]. 
type-(5) spinor field. It is worthwhile to point out a peculiar feature of types-(4), -(5) and -(6) spinor fields: although $\mathbf{J}$ is always non-zero, $\mathbf{J}^{2}=-\mathbf{K}^{2}=0$. It shall be seen below that the bilinear covariants related to an ELKO spinor field, satisfy $\sigma=0=\omega, \mathbf{K}=0, \quad \mathbf{S} \neq 0$ and $\mathbf{J}^{2}=0$. Since Lounesto proved that there are no other classes based on distinctions among bilinear covariants, ELKO spinor fields must belong to one of the disjoint six classes.

Types-(1), -(2) and -(3) Dirac spinor fields (DSFs) have different algebraic and geometrical characters, and we would like to emphasize the main differing points. For more details, see e.g. 6, 7]. Recall that if the quantities $P=\sigma+\mathbf{J}+\gamma_{0123} \omega$ and $Q=\mathbf{S}+\mathbf{K} \gamma_{0123}$ are defined [6, 7], in type-(1) DSF we have $P=-\left(\omega+\sigma \gamma_{0123}\right)^{-1} \mathbf{K} Q$ and also $\psi=-i\left(\omega+\sigma \gamma_{0123}\right)^{-1} \psi$. In type-(2) DSF, $P$ is a multiple of $\frac{1}{2 \sigma}(\sigma+\mathbf{J})$ and looks like a proper energy projection operator, commuting with the spin projector operator given by $\frac{1}{2}\left(1-i \gamma_{0123} \mathbf{K} / \sigma\right)$. Also, $P=\gamma_{0123} \mathbf{K} Q / \sigma$. Further, in type-(3) DSF, $P^{2}=0$ and $P=\mathbf{K} Q / \omega$. The introduction of the spin-Clifford bundle makes it possible to consider all the geometric and algebraic objects - the Clifford bundle, spinor fields, differential form fields, operators and Clifford fields — as being elements of an unique unified formalism. It is well known that spinor fields have three different, although equivalent, definitions: the operatorial, the classical and the algebraic one. In particular, the operatorial definition allows us to factor - up to sign - the DSF $\psi$ as $\psi=\left(\sigma+\omega \gamma_{0123}\right)^{-1 / 2} R$, where $R \in \operatorname{Spin}_{1,3}^{e}$. Denoting $\mathbf{K}_{k}=\psi \gamma_{k} \tilde{\psi}$, where $\tilde{\psi}$ denotes the reversion of $\psi$, the set $\left\{\mathbf{J}, K_{1}, K_{2}, K_{3}\right\}$ is an orthogonal basis of $\mathbb{R}^{1,3}$. On the other hand, in classes (4), (5) and (6) - where $\sigma=\bar{\psi} \psi=0=\omega=\bar{\psi} \gamma_{5} \psi$, the vectors $\left\{\mathbf{J}, K_{1}, K_{2}, K_{3}\right\}$ no longer form a basis and collapse into a null-line [6, 7]. In such case only the boundary term is non null. Finally, to a Weyl spinor field $\xi$ (type-(6)) with bilinear covariants $\mathbf{J}$ and $\mathbf{K}$, two Majorana spinor fields $\psi_{ \pm}=\frac{1}{2}(\xi+C(\xi))$ can be associated, where $C$ denotes the charge conjugation operator. Penrose flagpoles are implicitly defined by the equation $\sigma+\mathbf{J}+i \mathbf{S}-i \gamma_{0123} \mathbf{K}+\gamma_{0123} \omega=\frac{1}{2}\left(\mathbf{J} \mp i \mathbf{S} \gamma_{0123}\right)[6$, 7]. For a physically useful discussion regarding the disjoint classes -(5) and -(6) see, e.g., [20]. The fact that two Majorana spinor fields $\psi_{ \pm}$can be written in terms of a Weyl type-(6) spinor field $\psi_{ \pm}=\frac{1}{2}(\xi+C(\xi))$, is an 'accident' when the (Lorentzian) spacetime has $n=4$ - the present case - or $n=6$ dimensions. The more general assertion concerns the property that two Majorana, and more generally ELKO spinor fields $\psi_{ \pm}$ can be written in terms of a pure spinor field - hereon denoted by $\mathfrak{u}$ - as $\psi_{ \pm}=\frac{1}{2}(\mathfrak{u}+C(\mathfrak{u}))$. It is well known that Weyl spinor fields are pure spinor fields when $n=4$ and $n=6$. When the complexification of $\mathbb{C} \otimes \mathbb{R}^{1,3}$ of $\mathbb{R}^{1,3}$ is considered, one can consider a maximal totally isotropic subspace $N$ of $\mathbb{C}^{1,3}$, by the Witt decomposition, where $\operatorname{dim}_{\mathbb{C}} N=2$. Pure spinors are defined by the property $x \mathfrak{u}=0$ for all $x \in N \subset \mathbb{C}^{1,3}$. In this context, Penrose flags can be defined by the 
expression $\operatorname{Re}(i \mathfrak{u} \tilde{\mathfrak{u}})$.

[1] D. V. Ahluwalia-Khalilova and D. Grumiller, Spin Half Fermions, with Mass Dimension One: Theory, Phenomenology, and Dark Matter, JCAP 07 (2005) 012 [arXiv:hep-th/0412080v3].

[2] D. V. Ahluwalia-Khalilova and D. Grumiller, Dark matter: A spin one half fermion field with mass dimension one?, Phys. Rev. D72 (2005) 067701 arXiv:hep-th/0410192v2].

[3] D. V. Ahluwalia, Cheng-Yang Lee, D. Schritt, T. F. Watson, Dark matter and dark gauge fields, in "Dark matter in astroparticle and particle physics, DARK 2007, Proceedings of the 6th international Heidelberg conference" (24-28 September 2007, Sydney, Australia), Eds. H. V. Klapdor-Kleingrothaus and G. F. Lewis, pp. 198-208. arXiv:0712.4190v2 [hep-ph]]; Local fermionic dark matter with mass dimension one, [arXiv:0804.1854v4 [hep-th]].

[4] R. da Rocha and J. M. Hoff da Silva, From Dirac spinor fields to eigenspinoren des ladungskonjugationsoperators, J. Math. Phys. 48 (2007) 123517 [arXiv:0711.1103 [math-ph]].

[5] R. da Rocha and W. A. Rodrigues, Jr., Where are ELKO spinor fields in Lounesto spinor field classification?, Mod. Phys. Lett. A21 (2006) 65-74 [arXiv:math-ph/0506075v3].

[6] P. Lounesto, Clifford Algebras, Relativity and Quantum Mechanics, in P. Letelier and W. A. Rodrigues, Jr. (eds.), Gravitation: the Spacetime Structure, Proc. of the $8^{\text {th }}$ Latin American Symposium on Relativity and Gravitation, Águas de Lindóia, Brazil, 25-30 July 1993, World-Scientific, London 1993.

[7] P. Lounesto, Clifford Algebras and Spinors, $2^{\text {nd }}$ ed., pp. 152-173, Cambridge Univ. Press, Cambridge 2002.

[8] D. V. Ahluwalia-Khalilova, Dark matter, and its darkness, Int. J. Mod. Phys. D15 (2006) 2267-2278 arxiv:hep-th/0603545v3].

[9] C. G. Boehmer, The Einstein-Elko system - Can dark matter drive inflation?, Annalen Phys. 16 (2007) 325-341 arXiv:gr-qc/0701087v1]; The Einstein-Cartan-Elko system, Annalen Phys. 16 (2007) 38-44 [arXiv:gr-qc/0607088v1]; Dark spinor inflation - theory primer and dynamics, Phys. Rev. D 77 (2008) 123535 [arXiv:0804.0616v1 [astro-ph]].

[10] R. da Rocha and J. M. Hoff da Silva, ELKO, flagpole and flag-dipole spinor fields, and the instanton Hopf fibration, accepted for publication in Adv. Appl. Clifford Alg. (2009) [arXiv:0811.2717v1 [math-ph]].

[11] R. A. Mosna and W. A. Rodrigues, Jr., The bundles of algebraic and Dirac-Hestenes spinor fields, J. Math. Phys. 45 (2004) 2945-2988 [arXiv:math-ph/0212033v5].

[12] R. da Rocha and J. M. Hoff da Silva, ELKO Spinor Fields: Lagrangians for Gravity derived from Supergravity, accepted for publication in Int. J. Geom. Meth. Mod. Phys. (2009) arXiv:0901.0883v1 [math-ph]].

[13] R. S. Tung, Gravitation as a supersymmetric gauge theory, Phys. Lett. A 264 (2000) 341-345 arXiv:gr-qc/9904008. 
[14] R. da Rocha R and J. G. Pereira, The quadratic spinor Lagrangian, axial torsion current, and generalizations, Int. J. Mod. Phys. D 16 (2007) 1653-1667 [arXiv:gr-qc/0703076v1].

[15] W. A. Rodrigues, Jr., Algebraic and Dirac Hestenes Spinors and Spinor Fields, J. Math. Phys. 45 (2004) 2908-2966 [arXiv:math-ph/0212030v6.]

[16] J. P. Crawford, On the Algebra of Dirac Bispinor Densities: Factorization and Inversion Theorems, J. Math. Phys. 26 (1985) 1429-1441; The geometric structure of the space of fermionic physical observables, em Micali A et al.(eds.) Clifford Algebras and Their Applications in Math. Physics, Kluwer Acad. Publishers, Dordrecht 1989.

[17] P. R. Holland, Relativistic Algebraic Spinors and Quantum Motions in Phase Space, Found. Phys. 16 (1986) 708-709.

[18] P. R. Holland, Minimal Ideals and Clifford Algebras in the Phase Space Representation of spin-1/2 Fields, p. 273-283 in Chisholm J S R and Common A K (eds.), Proceedings of the Workshop on Clifford Algebras and their Applications in Mathematical Physics (Canterbury 1985), Reidel, Dordrecht 1986.

[19] M. R. Francis and A. Kosowsky, The construction of spinors in geometric algebra, Annals Phys. 317 (2005) 383-409 [arXiv: math-ph/0403040v2].

[20] R. Plaga, The non-equivalence of Weyl and Majorana neutrinos with standard-model gauge interactions, arXiv:hep-ph/0108052v1]. 